\title{
DESCRIPTION OF THE 3D MORPHOLOGY OF GRAIN BOUNDARIES IN ALUMINUM ALLOYS USING TESSELLATION MODELS GENERATED BY ELLIPSOIDS
}

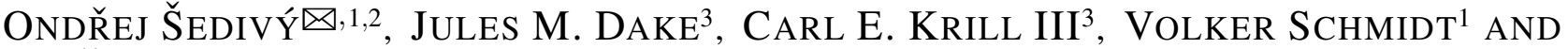 \\ ALEŠ J $̈ ̈ G E R{ }^{2}$ \\ ${ }^{1}$ Institute of Stochastics, Ulm University, Helmholtzstrasse 18, 89069 Ulm, Germany; ${ }^{2}$ Institute of Physics, \\ Czech Academy of Sciences, Na Slovance 1999/2, 18221 Prague, Czech Republic; ${ }^{3}$ Institute of Micro and \\ Nanomaterials, Ulm University, Albert-Einstein-Allee 47, 89081 Ulm, Germany \\ e-mail: ondrej.sedivy@uni-ulm.de,jules.dake@uni-ulm.de, carl.krill@uni-ulm.de,volker.schmidt@uni-ulm.de, \\ jager@fzu.cz \\ (Received November 1, 2016; revised February 3, 2017; accepted February 8, 2017)
}

\begin{abstract}
Parametric tessellation models are often used to approximate complex grain morphologies of polycrystalline microstructures. A big advantage of such models is the substantial reduction in disk space required to store large, three-dimensional data sets, especially when compared with voxel-based alternatives. By selection of an appropriate tessellation model, a reasonably small loss of information on the real grain shapes can usually be achieved. Special attention has recently been devoted to models based on ellipsoidal approximations fitted to each grain. Faces of these tessellations are portions of quadric surfaces whose parameters can be derived easily. In this paper, we deal with geometric features of the structure, notably curvatures and dihedral angles, which are closely related to the kinetics of grain growth. These characteristics are computed for ellipsoidbased tessellations fitted to two different aluminum alloys with nominal composition Al-3 wt $\% \mathrm{Mg}-0.2 \mathrm{wt} \% \mathrm{Sc}$ and $\mathrm{Al}-1 \mathrm{wt} \% \mathrm{Mg}$. The results are then compared with estimations based on meshed empirical data. We observe that the model offers more consistent estimations of grain shape characteristics than do the meshed empirical data. Precise description of grain boundaries by the model is also promising with respect to possible applications of these tessellations in stochastic space-time modeling of grain growth.
\end{abstract}

Keywords: curvature, dihedral angle, grain boundary, polycrystalline material, tessellation.

\section{INTRODUCTION}

The explanation of microstructural changes induced in polycrystalline metals by various kinds of thermomechanical treatments is a prerequisite for understanding their macroscopic behavior. We are particularly interested in modeling the spatial arrangements of grain boundaries in these materials, since the grain boundaries are of paramount importance in explaining many physical phenomena. Obviously, the morphology of grain boundaries is directly related to mechanical response, thermal and electrical conductivity, magnetism and many other properties of polycrystalline materials (Grimvall, 1999).

From a mathematical point of view, the grain microstructure of polycrystalline materials can be described by tessellations, as the latter are divisions of space into non-overlapping regions (Chiu et al., 2013). Many parametric tessellation models have been invented to describe cellular structures in various fields of research (Okabe et al., 2010). Concerning materials science, great interest has been devoted to the application of convex tessellation models, in which the grains are convex polyhedra; see, e.g., Lyckegaard et al. (2011). The simplicity of these models allows a simple evaluation of size and shape characteristics of the grains (Lautensack and Zuyev, 2008) and relatively fast and accurate fitting to empirical data (Spettl et al., 2016). However, in connection with recent progress in microscopic research, the interest of scientists has significantly increased regarding more general tessellations with curved boundaries, which can better describe real grain shapes.

In Altendorf et al. (2014), several such models were applied to martensitic and bainitic steels. These models are based on isotropic or anisotropic grain growth, where the tessellations are constructed on the basis of an initial approximation of the grains by balls or ellipsoids. Optimized methods for fitting these models to real data were presented in Alpers et al. (2015), Teferra and Graham-Brady (2015) and Šedivý et al. (2016). These results have shown that, in particular, the ellipsoid-based tessellation models are able to describe a great variety of grain microstructures with very high precision. 
With the advent of new tessellation models used in materials science, there is a need for thorough investigation of geometric properties of these models. Their simple parametric form often allows to express geometric characteristics by explicit formulas. Thus, as another benefit of these models, they can replace further processing of the data needed to obtain the smooth surfaces from which these characteristics are usually estimated.

The estimation of geometric characteristics from ellipsoid-based tessellation models is the main contribution of this paper. While previous research has mostly concentrated on representing real microstructures by these models, significantly fewer results are available concerning geometric characterization of the models. We show that size and shape characteristics, which are commonly used for the statistical description of this kind of data, can be analytically computed from the model parameters. At first, we focus on volumes, surface areas and lengths of the grains, grain boundaries and triple grain junctions, respectively. Further, we deal with the estimation of surface curvatures defined on grain boundaries and dihedral angles defined on triple junctions. We also provide a comparison with estimations by conventional methods from empirical data.

The aforementioned characteristics have a straightforward application in modeling real grain growth. The knowledge of mean curvature allows to predict the capillary-driven migration of grain boundaries, while dihedral angles are needed for the description of constraints at the triple junctions (Gottstein and Shvindlerman, 2010). The size characteristics are useful for quantification of the microstructural changes caused by the migration of grain boundaries.

\section{EXPERIMENTAL BACKGROUND}

We consider two experimental data sets obtained by different experimental techniques. Sample I is an aluminum alloy with nominal composition Al$3 \mathrm{wt} \% \mathrm{Mg}-0.2 \mathrm{wt} \% \mathrm{Sc}$. A sample with square cross section was processed by eight passes of equal channel angular pressing (ECAP) at room temperature and subsequently annealed for $1 \mathrm{~h}$ at $400^{\circ} \mathrm{C}$. These processes led to a microstructure with well-defined fine grains and with mean grain size of $1.9 \mu \mathrm{m}^{3}$; see Fig. 1a.

A 3D image of the microstructure of Sample I was obtained by electron backscatter diffraction (EBSD). In this method, the sample is repeatedly exposed to two different beams, an electron beam for scanning the surface of the sample and a focused ion beam for sectioning the material (Schwartz et al., 2009). In such a manner, a 3D image is obtained from a stack of 2D images. The size of the final 3D image, resulting from preprocessing and alignment of the section planes, was $21.6 \times 28.9 \times 12.0 \mu^{3}$ and the voxel size was $0.1^{3} \mu \mathrm{m}^{3}$. For more details on sample preparation and data acquisition, see Šedivý et al. (2016), Šedivý and Jäger (2017).

Sample II was taken from an aluminum alloy with nominal composition Al-1 wt\% Mg. A cylindrical specimen of the material was annealed at $400^{\circ} \mathrm{C}$ for $1 \mathrm{~h}$. This process led to a microstructure with mean grain size of $0.36 \mathrm{~mm}^{3}$; see Fig. $1 \mathrm{~b}$.

A 3D image of the microstructure of Sample II was obtained by 3D X-ray diffraction (3DXRD) microscopy. In this nondestructive technique, the material is illuminated with a monochromatic X-ray beam and rotated up to $360^{\circ}$, such that crystallographic information from the whole volume is obtained. The experiment was performed at the synchrotron radiation facility SPring-8. The final cylindrical data had an approximate radius of $0.8 \mathrm{~mm}$ and height of $2.7 \mathrm{~mm}$, and the voxel size was $5^{3} \mu \mathrm{m}^{3}$. Details on data acquisition and reconstruction can be found in Dake et al. (2016).

\section{TESSELLATION MODELS}

Consider a countable collection of closed sets, $\mathscr{T}=\left\{C_{i} \subset \mathbb{R}^{3}\right\}$, such that

1. $\stackrel{\circ}{C}_{i} \cap \stackrel{\circ}{C}_{j}=\emptyset$ for all $i \neq j$, where $\stackrel{\circ}{C}_{i}$ is the interior of the set $C_{i}$,

2. $\bigcup_{i} C_{i}=\mathbb{R}^{3}$,

3. $\mathscr{T}$ is locally finite, i.e., $\#\left\{C_{i} \in \mathscr{T}: C_{i} \cap B \neq \emptyset\right\}<\infty$ for all bounded $B \subset \mathbb{R}^{3}$.

Then $\mathscr{T}$ is called a tessellation of the space $\mathbb{R}^{3}$. A tessellation can be viewed as a division of space into non-overlapping sets, which are usually called cells or grains. For more details, see, e.g., Chiu et al. (2013).

In the following we focus on tessellations generated by a locally finite point pattern, $\mathscr{P}$, the points of which we call the seeds or generators of the tessellation. The cell $C_{i}$ corresponding to a given seed, $\mathbf{x}_{i} \in \mathscr{P}$, is defined as the set of points in $\mathbb{R}^{3}$ that are closer to $\mathbf{x}_{i}$ than to any other seed in $\mathscr{P}$ with respect to an appropriate distance measure $d$. Formally,

$$
C_{i}=\left\{\mathbf{y} \in \mathbb{R}^{3}: d\left(\mathbf{y}, \mathbf{x}_{i}\right) \leq d\left(\mathbf{y}, \mathbf{x}^{\prime}\right) \text { for all } \mathbf{x}^{\prime} \in \mathscr{P}\right\} .
$$




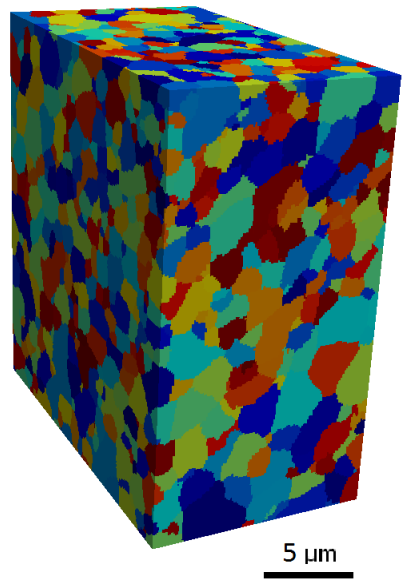

a)

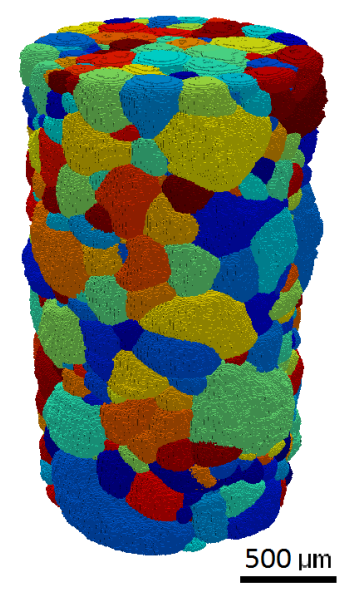

b)
Fig. 1. Grain microstructure of the analyzed samples. a) Sample of Al-3 wt\% Mg-0.2 wt\% Sc acquired by $3 D$ EBSD, $b$ ) sample of Al-1 wt\% $\mathrm{Mg}$ acquired by $3 D X R D$.

A classical tessellation model is the Voronoi tessellation, where the distance measure is given by the Euclidean distance

$$
d_{V}\left(\mathbf{y}, \mathbf{x}_{i}\right)=\left\|\mathbf{y}-\mathbf{x}_{i}\right\| .
$$

The cells of a Voronoi tessellation are convex polyhedra; see, e.g., Chiu et al. (2013). In more general cases, we furnish each generating point with additional parameters. Cells of the tessellation are then generated by a marked point pattern, and the distance measure $d$ in Eq. 1 is a function of both the points' locations and their additional parameters (marks). A first extension of the Voronoi tessellation is the Laguerre tessellation or power diagram; see Lautensack and Zuyev (2008). Its generating marked point pattern is $\mathscr{P}=\left\{\mathbf{x}_{i}, w_{i}\right\} \subseteq$ $\mathbb{R}^{3} \times \mathbb{R}$, and the distance measure is given as

$$
d_{L}\left(\mathbf{y},\left(\mathbf{x}_{i}, w_{i}\right)\right)=\left\|\mathbf{y}-\mathbf{x}_{i}\right\|^{2}-w_{i} .
$$

Here, a real parameter, called power, is assigned to each cell, affecting its size and shape. As for the Voronoi tessellation, this definition leads to planar cell facets. However, the additional parameter allows for the generation of tessellations with greater variation in terms of both cell size and shape than Voronoi tessellations (Jeulin, 2013; Teferra and Graham-Brady, 2015). Actually, any normal tessellation in 3D can be described by a Laguerre tessellation (Lautensack and Zuyev, 2008). Note that the function $d_{L}$ can take on negative values, which simplifies subsequent considerations. However, positive distances can easily be obtained simply by shifting all weights by an appropriate additive constant that is identical for each seed. This operation does not change the inequality in Eq. 1.
Voronoi and Laguerre tessellations have extensively been used in literature to model grain microstructures; see, e.g., Kumar and Kurtz (1994) and Lyckegaard et al. (2011). However, modern methods of microscopic research have reliably proven that the grain boundaries in real microstructures are not perfectly planar. This motivates us to apply the definition given in Eq. 1 with different kinds of distance measures such that the boundaries between the cells of tessellations are curved.

Examples of such models were presented in Jeulin (2013) and Altendorf et al. (2014). Here, the socalled local Voronoi tessellation is defined using a local metric

$$
d_{\mathrm{M}}\left(\mathbf{y},\left(\mathbf{x}_{i}, M_{i}\right)\right)=\left(\mathbf{y}-\mathbf{x}_{i}\right)^{\top} M_{i}\left(\mathbf{y}-\mathbf{x}_{i}\right),
$$

where $M_{i}$ is a real-valued positive definite $3 \times 3$ matrix. Each cell of this tessellation has 6 parameters, which can be identified with the 6 independent entries of $M_{i}$. In the special case in which $M_{i}$ is the identity matrix, this model corresponds to the Voronoi tessellation.

This local metric model is further extended in Alpers et al. (2015) to obtain the generalized balanced power diagram (GBPD). This is a tessellation generated by the local distance measure

$$
d_{\mathrm{GB}}\left(\mathbf{y},\left(\mathbf{x}_{i}, M_{i}, w_{i}\right)\right)=\left(\mathbf{y}-\mathbf{x}_{i}\right)^{\top} M_{i}\left(\mathbf{y}-\mathbf{x}_{i}\right)-w_{i},
$$

where $M_{i}$ is a real-valued positive definite matrix and $w_{i} \in \mathbb{R}$. In this model, each cell is defined in terms of 3 coordinates of the seed and 7 additional parameters. In the special case where $M_{i}$ is the identity matrix, this model corresponds to the Laguerre tessellation.

In what follows we will use tessellations obtained from the local metric $d_{\mathrm{GB}}$, being the most general representative of a class of models that we entitle ellipsoid-based tessellations. Obviously, the same principles apply for its submodels, including the one obtained from the local metric $d_{\mathrm{M}}$, potentially having the matrix $M_{i}$ in a special form such that the number of free parameters is smaller. Such special cases include tessellations generated by oblate/prolate ellipsoids or balls.

Tessellations fitted to empirical data will be restricted to a bounded spatial domain $W \subset \mathbb{R}^{3}$. Obviously, cells of such tessellations are bounded. However, our definition allows tessellations to include lower-dimensional or empty cells (cells with which no point in real space is associated, because the corresponding set $C_{i}$ defined in Eq. 1 is empty). These cases correspond to a situation in which an observed grain has no counterpart in the fitted tessellation model. Often, when a small and irregularly shaped grain is surrounded by larger grains, then, in the model, the region containing the small grain will be covered 
by tessellation cells fitted to the larger neighbours. Consequently, an empty cell is produced. A nonempty, but lower-dimensional cell can arise if the seed of the diminished cell lies exactly on a facet of the tessellation.

\section{MODEL FITTING}

Fitting of ellipsoid-based tessellations to real data is a challenging task. For larger data sets, the problem becomes high dimensional because the number of parameters in the presented models increases linearly with the number of grains. However, simple heuristic approaches exist that usually provide nice approximations (Altendorf et al., 2014). For ellipsoidbased tessellations, the heuristic approximation is based on best-fitting ellipsoids found to the original grains. The centres of ellipsoids are identified as centres of mass of the grains, and the principal axes are found by principal component analysis.

This heuristic approach can be improved further by optimization techniques that iteratively increase the accuracy of the fit by modifying the parameters. Such methods were presented, e.g., in Alpers et al. (2015); Teferra and Graham-Brady (2015). However, they are difficult to apply to large data sets. We rather use a stochastic optimization algorithm based on simulated annealing presented in Šedivý et al. (2016). In each step of this algorithm, only a subset of parameters of one grain is modified. This allows a fast reconstruction of the modified tessellation, because the change affects only the immediate vicinity of the modified grain. The suggested change in parameter values is accepted with a certain probability in each iteration, such that an optimal solution is approached.

To assess the quality of the fitted model with respect to empirical data, we utilize several criteria that compare the structures in both a metrical and a topological sense. Assume that the empirical image data are observed on a voxel grid $W^{\prime} \subset W$ within a bounded window $W \subset \mathbb{R}^{3}$. Let $I(\mathbf{x})$ denote the grain index at a point $\mathbf{x}$ in the empirical image data and $I_{\mathscr{T}}(\mathbf{x})$ the index of the cell of the tessellation fitted to this data. Further, by $B_{I}$ we denote the set of boundary voxels in the empirical image data, i.e.,

$$
B_{I}=\left\{\mathbf{x} \in W^{\prime}: \exists \mathbf{y} \in W^{\prime}, I(\mathbf{x}) \neq I(\mathbf{y}),\|\mathbf{x}-\mathbf{y}\| \leq \sqrt{3}\right\} .
$$

The latter condition (with distance measured in voxel size) expresses the requirement that the corresponding voxels represented as cubes share at least a point. By $B_{\mathscr{T}}$ we denote an analogous set of boundary voxels in the tessellation model. The basic discrepancy measure

$$
\mathscr{D}_{I, \mathscr{T}}=\frac{\#\left\{\mathbf{x} \in W^{\prime}: I(\mathbf{x}) \neq I_{\mathscr{T}}(\mathbf{x})\right\}}{\#\left\{\mathbf{x} \in W^{\prime}\right\}}
$$

provides information on the fraction of voxels for which these two indices are different. For a local quality of fit near the grain boundaries, we use the distance of the observed grain boundaries to their modeled counterparts. This distance is evaluated for each pair of neghbouring boundary points with different grain index, $\mathbf{x}, \mathbf{y} \in B_{I}: I(\mathbf{x}) \neq I(\mathbf{y}),\|\mathbf{x}-\mathbf{y}\| \leq$ $\sqrt{3}$, and it is computed as

$$
v_{I, \mathscr{T}}(\mathbf{x}, \mathbf{y})=\min _{\mathbf{z} \in B_{\mathscr{T}}^{*}(I(\mathbf{x}), I(\mathbf{y}))}\left\|\frac{\mathbf{x}+\mathbf{y}}{2}-\mathbf{z}\right\|,
$$

where $B_{\mathscr{T}}^{*}(i, j)$ stands for the exact boundary between cells $C_{i}$ and $C_{j}$ in the tessellation model.

Finally, we define some topological characteristics. Denote by $G_{i} \sim G_{j}$ the relation that the grains $G_{i}$ and $G_{j}$ are neighbours, i.e., there exists a pair of points $\mathbf{x}, \mathbf{y} \in B_{I}: I(\mathbf{x})=i, I(\mathbf{y})=j,\|\mathbf{x}-\mathbf{y}\| \leq \sqrt{3}$. Analogously, $C_{i} \sim C_{j}$ denotes the neighbourhood relationship of cells in the fitted tessellation. Let $N$ be the total number of grains observed in the empirical image data. Then for the $i$ th grain the quantity

$$
\begin{array}{r}
\mu_{I, \mathscr{T}}(i)=\#\left\{j \in 1, \ldots, N:\left(G_{i} \sim G_{j} \text { and } C_{i} \not C_{j}\right)\right. \\
\text { or } \left.\left(G_{i} \not G_{j} \text { and } C_{i} \sim C_{j}\right)\right\}
\end{array}
$$

describes the neighbourhood fit by counting the number of disagreements in the list of neighbours in the observed data and the model, respectively. The average

$$
\bar{\mu}_{I, \mathscr{T}}=\frac{1}{N} \sum_{i=1}^{N} \mu_{I, \mathscr{T}}(i)
$$

is called the mean neighbourhood fit.

\section{SIZE CHARACTERISTICS}

The boundaries between cells in the ellipsoidbased model are parts of quadric surfaces. Using the parameters from Eq. 5, these quadrics can be represented as

$$
Q_{i j}(\mathbf{x})=\mathbf{x}^{\top} A_{i j} \mathbf{x}+B_{i j} \mathbf{x}+C_{i j}=0,
$$

where

$$
\begin{aligned}
A_{i j} & =M_{i}-M_{j}, \\
B_{i j} & =-2\left(M_{i} \mathbf{x}_{i}-M_{j} \mathbf{x}_{j}\right), \\
C_{i j} & =\mathbf{x}_{i}^{\top} M_{i} \mathbf{x}_{i}-\mathbf{x}_{j}^{\top} M_{j} \mathbf{x}_{j} .
\end{aligned}
$$


This implicit representation can be transformed to a parametric representation of the surface

$$
q_{i j}(u, v)=(x(u, v), y(u, v), z(u, v)), \quad u, v \in \mathscr{S} \subset \mathbb{R}^{2},
$$

and, similarly, for triple grain junctions we can obtain a parametric representation of the curve

$$
t_{i j k}(u)=(x(u), y(u), z(u)), \quad u \in \mathscr{L} \subset \mathbb{R} .
$$

Here, the sets $\mathscr{S}$ and $\mathscr{L}$ represent the restricted domains for the parameters such that the bounded surfaces or curves are generated. For the parametrization, a general method can be used that is based on minimal $\mu$-bases (Chen et al., 2007). Both parametric functions $q_{i j}(u, v)$ and $t_{i j k}(u)$ are differentiable in each inner point of the boundary or the triple junction, respectively, because they are represented by polynomial equations. Using the aforementioned representations, the basic size characteristics can be expressed as follows. The volume of a grain $G_{i}$ is given by

$$
V_{i}=\int_{\mathbf{x} \in G_{i}} \mathrm{~d} \mathbf{x}
$$

the surface area of a boundary between grains $G_{i}$ and $G_{j}$ is given by

$$
S_{i j}=\int_{\mathscr{S}}\left\|\frac{\partial q_{i j}(u, v)}{\partial u} \times \frac{\partial q_{i j}(u, v)}{\partial v}\right\| \mathrm{d}(u, v),
$$

and the length of a triple junction shared by the grains $G_{i}, G_{j}$ and $G_{k}$ reads

$$
L_{i j k}=\int_{\mathscr{L}}\left\|\frac{\partial t_{i j k}(u)}{\partial u}\right\| \mathrm{d} u .
$$

\section{LOCAL SHAPE CHARACTERISTICS}

Consider a point $\mathbf{x}$ lying at a triple junction shared by the grains $G_{i}, G_{j}$ and $G_{k}$. The normal vectors to the three boundary surfaces are

$$
\begin{aligned}
\mathbf{n}_{i j}(\mathbf{x}) & = \pm \nabla Q_{i j}(\mathbf{x}), \\
\mathbf{n}_{i k}(\mathbf{x}) & = \pm \nabla Q_{i k}(\mathbf{x}), \\
\mathbf{n}_{j k}(\mathbf{x}) & = \pm \nabla Q_{j k}(\mathbf{x}) .
\end{aligned}
$$

With appropriate signs for the normal vectors, the dihedral angles can be evaluated as

$$
\alpha_{i j}(\mathbf{x})=\arccos \left(1-\frac{\left\|\tilde{\mathbf{n}}_{i k}(\mathbf{x})-\tilde{\mathbf{n}}_{j k}(\mathbf{x})\right\|^{2}}{2}\right),
$$

etc., where $\tilde{\mathbf{n}}_{i j}(\mathbf{x})=\frac{\mathbf{n}_{i j}(\mathbf{x})}{\left\|\mathbf{n}_{i j}(\mathbf{x})\right\|}$.

To determine the curvature of the surface at a surface point $\mathbf{x}$, we consider the intersection curves of the surface with the planes orthogonal to the surface at point $\mathbf{x}$. Principal curvatures $\kappa_{1}(\mathbf{x}), \kappa_{2}(\mathbf{x})$ are defined as maximum and minimum curvature of the intersection curves at $\mathbf{x}$, the mean curvature is $\kappa_{M}(\mathbf{x})=$ $\left(\kappa_{1}(\mathbf{x})+\kappa_{2}(\mathbf{x})\right) / 2$, and the Gaussian curvature is $\kappa_{G}(\mathbf{x})=\kappa_{1}(\mathbf{x}) \kappa_{2}(\mathbf{x})$.

For the implicit surface given by the equation $Q(\mathbf{x})=0$, we denote the Hessian by $H(\mathbf{x})$, and the matrix of its cofactors by $H^{*}(\mathbf{x})$. Then the Gaussian curvature can be expressed as

$$
\kappa_{G}(\mathbf{x})=\frac{\nabla Q(\mathbf{x}) H^{*}(\mathbf{x}) \nabla Q(\mathbf{x})^{\top}}{\|\nabla Q(\mathbf{x})\|^{4}},
$$

the mean curvature is

$$
\kappa_{M}(\mathbf{x})=\frac{\nabla Q(\mathbf{x}) H(\mathbf{x}) \nabla Q(\mathbf{x})^{\top}-\nabla Q(\mathbf{x})^{2} \operatorname{tr}(H(\mathbf{x}))}{2\|\nabla Q(\mathbf{x})\|^{3}},
$$

where $\operatorname{tr}(\cdot)$ is the trace of a matrix, and the principal curvatures are

$$
\kappa_{1,2}=\kappa_{M}(\mathbf{x}) \pm \sqrt{\kappa_{M}(\mathbf{x})^{2}-\kappa_{G}(\mathbf{x})},
$$

see Goldman (2005).

Finally, we explain how the same characteristics were computed from the empirical data. The grain volumes were simply computed by counting the voxels belonging to particular grains. For the other characteristics, the voxelized grain boundaries were first transformed to fine triangular surface meshes, using the filters 'Quick Surface Mesh' and 'Laplacian Smoothing' from the DREAM.3D software library (Groeber and Jackson, 2014). Grain boundary surfaces were obtained by summing up the areas of the triangles of these meshes that correspond to given boundaries. Similarly, the lengths of triple junctions were obtained by summing up the lengths of the triangle edges that correspond to these junctions.

The triangular surface meshes were also used for computing the curvatures. However, the accuracy is not sufficient near triple junctions. Indeed, during the Laplacian smoothing, triangles lying in the immediate vicinity of the junction are influenced by the vertices of triangles lying on adjacent boundaries. For this reason, the curvatures were evaluated only on triangles located at a sufficient distance from the triple junctions.

A similar difficulty arises with the dihedral angles, which are to be evaluated from the normal vectors at triple junctions, see Eq. 14. However, the normal 
vectors can hardly be estimated near triple junctions with sufficient accuracy. This can be overcome by computing the normal vectors at a certain distance from the junction, where the influence of adjacent boundaries on the surface mesh is negligible.

\section{RESULTS}

Both experimental data sets were approximated with ellipsoid-based tessellations using the simulated annealing methodology described in Šedivý et al. (2016). With a stopping condition defined therein, the algorithm was terminated after roughly 22 million iterations for Sample I and 5 million for Sample II, respectively, when no improvement in the fit was observed within a certain number of consecutive iterations. Table 1 summarizes the basic characteristics for evaluating the goodness of fit. From these results we see that the model provides a very good approximation to the empirical data. Expressed by the percentage of correctly assigned voxels, the fit is approximately $92 \%$ for Sample I and $95 \%$ for Sample II.

In addition, we assess the quality of the model by the functional characteristic given in Eq. 7. The latter offers better insight into local discrepancies between the observed grain boundaries and interfaces between cells in the fitted tessellation. The results of this comparison, depicted in Fig. 2, reveal that, for the majority of testing points on observed grain boundaries, the distance to the nearest interface in the model is within one voxel in both samples.

Concerning the size characteristics, we present results for grain volumes and surface areas of grain boundaries in Fig. 3. For the empirical data, these characteristics were computed from voxelized data (volumes) or meshed data (surface areas). For the model data, the characteristics were computed by numerical integration of the analytical formulas, see Eqs. 11, 12 and 13. Despite the fact that we deal with two different materials observed at very different length scales, the plots in Fig. 3 reveal a strong similarity in the distributions of the size characteristics. Moreover, the difference between estimation based on empirical data and computed from model data is very small.

Further, we focus on dihedral angles. For both empirical and model data, these angles were computed on a set of testing points on the triple grain boundary junctions. For each point, we have a triplet of angles summing up to $360^{\circ}$. The distributions of dihedral angles are compared in Fig. 4a. Dihedral angles measured from empirical data have a sharper peak about an equilibrating value of $120^{\circ}$. This result is to be expected, as quadric surfaces are less accurate in modeling real grain boundaries near triple junctions, where the boundaries are typically more highly curved due to specific constraints.

Table 1. Statistics for the quality of fit of approximations to empirical data by ellipsoidbased tessellations generated by the metric $d_{\mathrm{GB}}$. 1st row: percentage of correctly assigned voxels; 2 nd-4th row: percentage of cells with at most $0 / 1 / 2$ incorrect neighbours, respectively; 5th row: mean number of incorrect neighbours per grain.

\begin{tabular}{lrr}
\hline & Sample I & Sample II \\
\hline$\left(1-\mathscr{D}_{I, \mathscr{T}}\right) \cdot 100 \%$ & 92.03 & 95.03 \\
$\%$ of cells with $\mu_{I, \mathscr{T}}(\cdot)=0$ & 35.67 & 49.48 \\
$\%$ of cells with $\mu_{I, \mathscr{T}}(\cdot) \leq 1$ & 70.54 & 80.59 \\
$\%$ of cells with $\mu_{I, \mathscr{T}}(\cdot) \leq 2$ & 87.59 & 94.67 \\
$\bar{\mu}_{I, \mathscr{T}}$ & 1.20 & 0.79 \\
\hline
\end{tabular}

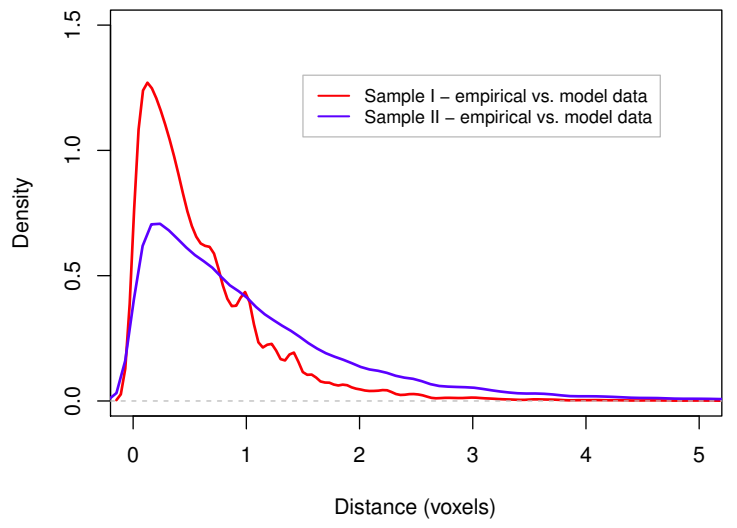

Fig. 2. Empirical distribution of the nearest distance from testing points on observed grain boundaries to the modeled grain boundaries.

Finally, we present results for curvatures. Similar to dihedral angles, curvatures were computed on a set of testing points on the grain boundaries. In Fig. $4 \mathrm{~b}$ we present the results for the Gaussian curvature. Because the grain boundaries are nearly planar, most of the curvatures are concentrated around zero. For better readability of the plots, we include only those values that exceed a threshold of 0.01 (in absolute value). Such a step has no effect other than lowering the peak near zero arising from the kernel estimation of the density. 


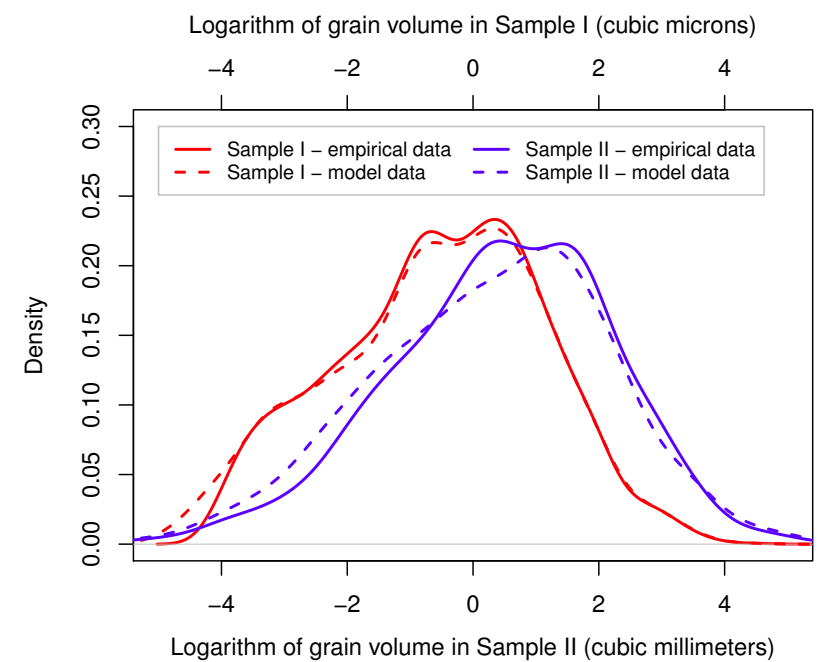

a)

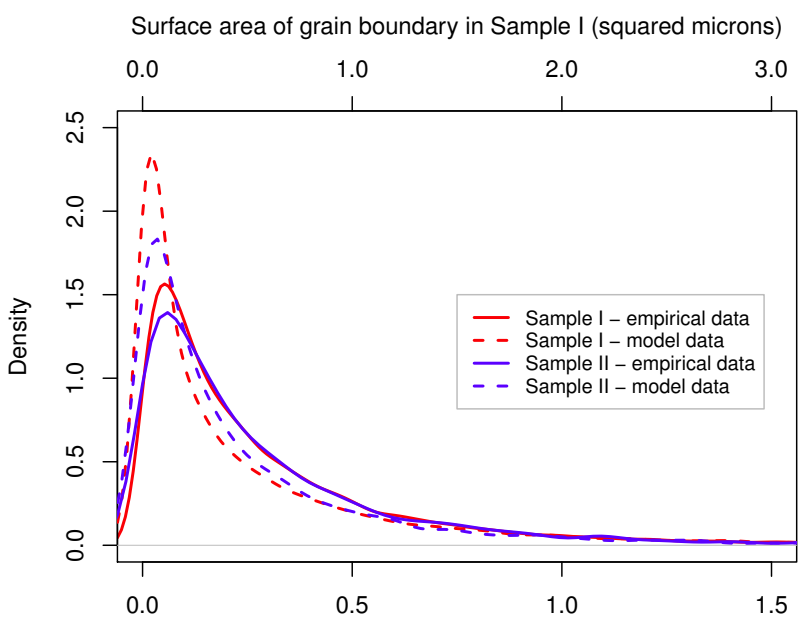

Surface area of grain boundary in Sample II (squared millimeters)

b)

Fig. 3. Comparison of the size characteristics in the empirical and the model data. a) Grain volume, b) surface area of grain boundary.

A typical feature of the empirical estimation is that negative values prevail. This is a consequence of the nature of the surface meshing procedure, in which an initial mesh is produced by drawing triangles on the voxel faces, and this mesh is iteratively smoothed. On the contrary, Gaussian curvatures evaluated from the model data are rather slightly positive, which is typical for the smooth quadric surfaces generated by the model. This is especially obvious for Sample II, where the majority of values lie within a short range centered about a positive value. A graphical representation of the curvatures on a subset of the empirical data is given in Fig. 5.

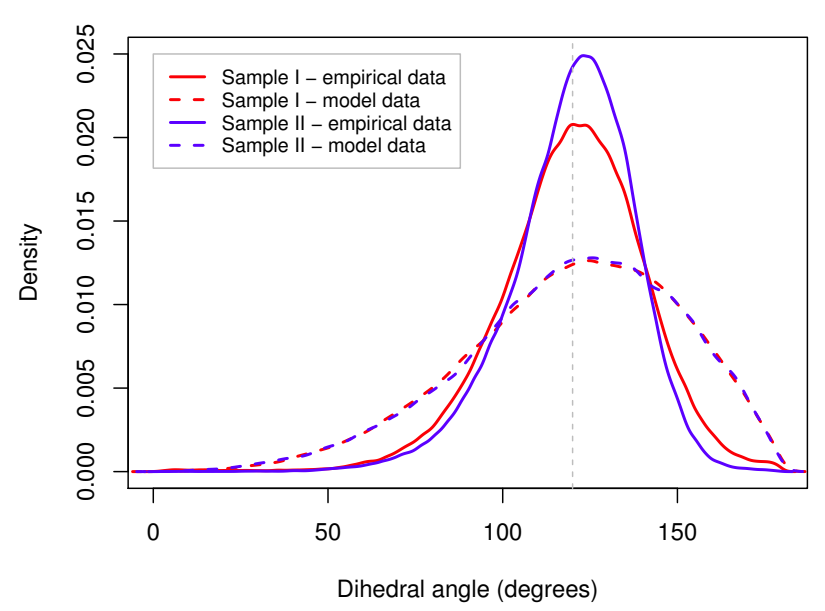

a)

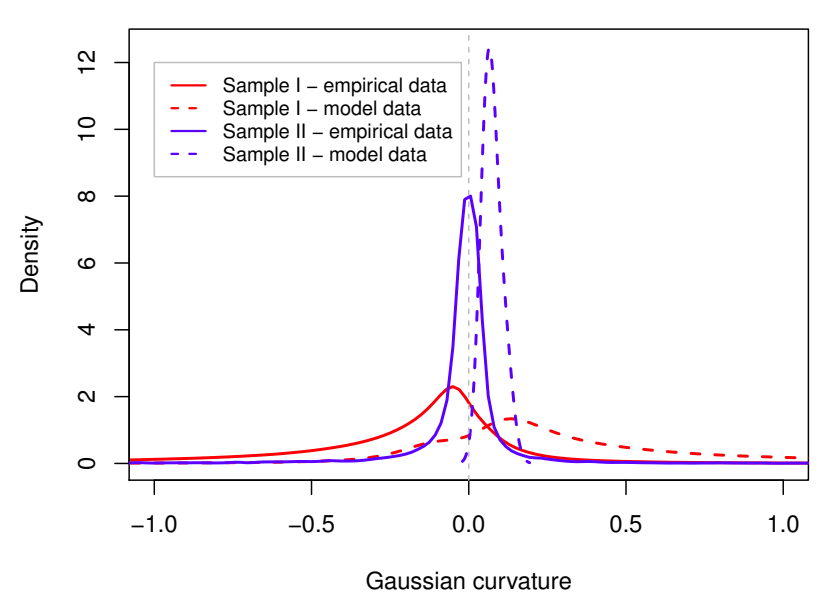

b)

Fig. 4. Comparison of the local shape characteristics of the empirical and model data. a) Dihedral angle, b) Gaussian curvature. Dashed vertical line stands for an equilibrating value of $120^{\circ}$ in subfigure (a) and zero Gaussian curvature in subfigure (b), respectively.

\section{CONCLUSIONS}

Our results confirm that ellipsoid-based tessellations are quite precise in describing polycrystalline microstructures. In particular, grain boundaries can be very well fitted by quadric surfaces, which arise as interfaces between cells in these models. Besides the commonly used discrepancy measures (Table 1), we support this claim by a functional characteristic that directly measures distances between the empirical and modeled grain boundaries on a dense set of testing points (Fig. 2). 


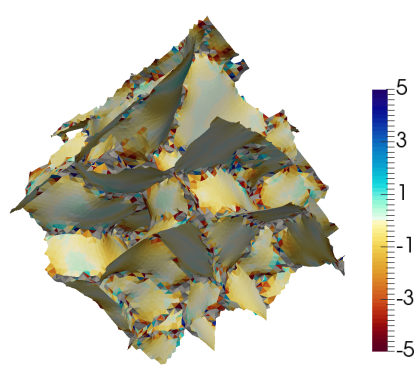

a)

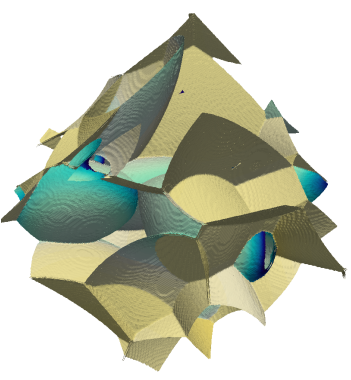

b)
Fig. 5. Gaussian curvature $\kappa_{M}(\mathbf{x})$ of grain boundaries in a cutout of Sample I. a) Empirical estimation based on a triangular surface mesh, b) estimation obtained from the ellipsoid-based tessellation model. Extreme curvatures near triple junctions in the empirical estimation are coloured gray.

A simple description of the modeled grain boundaries by quadric surfaces allows a tractable evaluation of size and shape characteristics of the grains, grain boundaries and triple junctions. Our results suggest that there is very good agreement between characteristics computed from both modeled and empirical image data. A great benefit of the model is that it can substitute the pre-processing operations that are required to obtain smooth grain boundaries. Moreover, the quadric surfaces resulting from the model are much more suitable for obtaining nice and consistent approximations of curvatures and dihedral angles between grain boundaries in comparison to estimations from meshed data, for which the smoothing parameters are difficult to calibrate properly.

\section{ACKNOWLEDGEMENTS}

This research was partially financed by the project GBP108/12/G043, Czech Science Foundation. We thank Leoš Polívka of the Institute of Physics of the Czech Academy of Sciences for the preparation of Sample I and 3D EBSD data acquisition. In addition, we are grateful to the Japan Synchrotron Radiation Research Institute for the allotment of beam time on beamline BL20XU of SPring-8 (Proposal 2013A1506), and we thank Dmitri Molodov of the Institute of Physical Metallurgy and Metal Physics, RWTH Aachen, for providing the Al- $1 \mathrm{wt} \% \mathrm{Mg}$ specimen.

\section{REFERENCES}

Alpers A, Brieden A, Gritzmann P, Lyckegaard A, Poulsen

HF (2015). Generalized balanced power diagrams for 3D representations of polycrystals. Phil Mag 95(9):1016-28.
Altendorf H, Latourte F, Jeulin D, Faessel M, Saintoyant L (2015). 3D reconstruction of a multiscale microstructure by anisotropic tessellation models. Image Anal Stereol 33(2):121-30.

Chen F, Shen L, Deng J (2007). Implicitization and parametrization of quadratic and cubic surfaces by $\mu$ bases. Computing 79:131-42.

Chiu SN, Stoyan D, Kendall WS, Mecke J (2013). Stochastic Geometry and its Applications. 3rd Ed. Chichester: Wiley.

Groeber MA, Jackson MA (2014). DREAM.3D: A digital representation environment for the analysis of microstructure in 3D. Integrat Mater Manuf Innov 3(1):1-17.

Dake JM, Oddershede J, Sørensen H, Werz T, Shatto JC, Uesegi K, Schmidt S, Krill III CE (2016). Direct observation of grain rotations during coarsening of a semisolid Al-Cu alloy. Proc Natl Acad Sci 113:E5998E6006.

Goldman R (2005). Curvature formulas for implicit curves and surfaces. Comput Aided Geom D 22:632-58.

Gottstein G, Shvindlerman LS (2010). Grain Boundary Migration in Metals. 2nd Ed. Boca Raton: CRC Press.

Grimvall G (1999). Thermophysical Properties of Materials. Amsterdam: Elsevier.

Jeulin D (2013). Random tessellations and Boolean random functions. In: Luengo Hendriks CL, Borgefors G, Strand R, Eds. Mathematical Morphology and Its Applications to Signal and Image Processing. Lect Not Comput Sci 7883:25-36.

Kumar S, Kurtz SK (1994). Simulation of material microstructure using a 3D Voronoi tesselation: Calculation of effective thermal expansion coefficient of polycrystalline materials. Acta Metall Mater 42(12):3917-27.

Lautensack C, Zuyev S (2008). Random Laguerre tessellations. Adv Appl Probab 40(3):630-50.

Lobkovsky AE, Karma A, Mendelev MI, Haataja M, Srolovitz DJ (2003). Grain shape, grain boundary mobility and the Herring relation. Acta Mater 52:28592.

Lyckegaard A, Lauridsen EM, Ludwig W, Fonda RW, Poulsen HF (2011). On the use of Laguerre tessellations for representations of 3D grain structures. Adv Eng Mater 13(3):165-70.

Okabe A, Boots B, Sugihara K, Chiu SN (2010). Spatial Tessellations: Concepts and Applications of Voronoi Diagrams. 2nd Ed. Chichester: Wiley.

Schwartz AJ, Kumar M, Adams BL, Field DP, Eds. (2009). Electron Backscatter Diffraction in Materials Science, 2nd Ed. New York: Springer. 
Šedivý O, Brereton T, Westhoff D, Polívka L, Beneš V, Schmidt V, Jäger A (2016). 3D reconstruction of grains in polycrystalline materials using a tessellation model with curved grain boundaries. Phil Mag 96(18):192649.

Šedivý O, Jäger A (2017). On correction of translational misalignments between section planes in 3D EBSD. J Microsc doi:10.1111/jmi.12528.

Spettl A, Brereton T, Duan Q, Werz T, Krill III CE, Kroese
DP, Schmidt V (2016). Fitting Laguerre tessellation approximations to tomographic image data. Phil Mag 96(2):166-89.

Teferra K, Graham-Brady L (2015). Tessellation growth models for polycrystalline microstructures. Comp Mater Sci 102:57-67.

Valiev RZ, Langdon TG (2006). Principles of equalchannel angular pressing as a processing tool for grain refinement. Prog Mater Sci 51(7):881-981. 